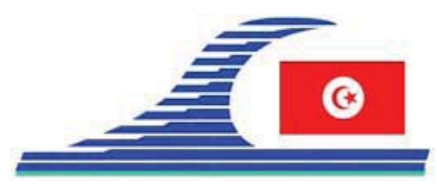

Conférence Méditerranéenne Côtière et Maritime EDITION 1, HAMMAMET, TUNISIE (2009)

Coastal and Maritime Mediterranean Conference

Disponible en ligne - http://www.paralia.fr-Available online

\title{
Impact sur l'environnement marin du golfe de Fos des rejets thermiques du CPT de Martigues
}

\section{Olivier BERTRAND ${ }^{1}$, Marie-Laure GENCO ${ }^{2}$, Philippe BORNENS ${ }^{3}$, Guillaume CARON ${ }^{4}$}

1. SOGREAH, Branche Eau Energie Environnement, Service Modélisation Hydraulique et Logiciels, 6 rue de Lorraine, 38130 Echirolles, France. olivier.bertrand@sogreah.fr

2. SOGREAH, Branche Maritime, Service Environnement Littoral, 6 rue de Lorraine, 38130 Echirolles, France. marie.laure.genco@sogreah.fr

3. IN VIVO, ZA la Grande Halte, 29940 La Forêt-Fouesnant, France. philippe.bornens@invivo-environnement.com

4. EDF CIT, Groupe Environnement, Tour Cèdre, 7 allée de l'Arche, TSA 31000, 92099 Paris la Défense Cedex, France.

guillaume-c.caron@edf.fr

\section{Résumé :}

Pour répondre à la demande d'électricité d'une région globalement déficitaire, EDF a pour projet de convertir deux des quatre tranches actuelles de la centrale fioul de Martigues Ponteau en cycle combiné gaz. Ce projet entre dans le champ d'application des lois sur l'eau et des Installations Classées pour la Protection de l'Environnement (ICPE). L'article décrit les études réalisées et les moyens mis en œuvre pour aboutir à un projet qui réponde aux objectifs d'exploitation en respectant au mieux les contraintes environnementales. Ces études consistent en une analyse de l'état actuel et une description multicritère du milieu marin devant servir de référence à l'évaluation de l'impact du projet et en la mise en place d'un modèle numérique destiné à évaluer l'impact sur le milieu des effluents du circuit de refroidissement de la centrale.

\section{Mots-clés :}

Golf de Fos - ICPE - Rejet thermique - Etude d'impact - Modélisation - Ecosystèmes côtiers

\section{Introduction}

EDF doit disposer de moyens de production permettant de faire face aux aléas de consommation. Aussi, et pour répondre aux ajustements de la consommation d'électricité, avec la souplesse d'utilisation que cela requiert aux moyens de production mobilisés, les centrales thermiques sont les solutions technico-économiques les plus souples pour répondre à ces aléas.

DOI: $10.5150 / \mathrm{cmcm} .2009 .043-5$ 
$\mathrm{Au}$ niveau environnemental, un Cycle Combiné Gaz (CCG) présente un bilan satisfaisant comparé aux autres filières à niveau de puissance et de production équivalentes. Aucune filière " fioul lourd » ne peut atteindre un niveau de rendement comparable à celui des Cycles Combinés.

Le Centre de Production Thermique (CPT) de Martigues Ponteau est implanté depuis le début des années 70 en bord de mer, à la frontière des zones industrielles du bassin de Fos et du littoral touristique de la côte bleue. Il est une composante essentielle du système de production électrique régional. EDF a pour projet de convertir deux des quatre tranches actuelles de la centrale fioul de Martigues en CCG, permettant ainsi de répondre à la demande d'électricité d'une région globalement déficitaire.

Le projet de Martigues entre dans le champ d'application des lois sur l'eau et des Installations Classées pour la Protection de l'Environnement (ICPE). En ce sens, l'étude d'impact du projet sur l'environnement marin doit être étudié avec comme préambule une analyse de l'état initial.

Nous décrivons ci-dessous les études réalisées et les moyens mis en œuvre pour aboutir à un projet qui réponde aux objectifs d'exploitation en respectant au mieux les contraintes environnementales.

\section{Etude de l'état initial}

Les moyens pour qualifier l'état initial reposent sur des campagnes de mesures, réalisées par IN VIVO, et sur une modélisation de la courantologie, mise au point par SOGREAH, permettant d'appréhender le milieu marin à l'échelle saisonnière.

Pour affiner la connaissance de la bathymétrie actuelle de la zone, des levés des fonds autour de la centrale ont été réalisés. Ces levés présentent un grand intérêt pour l'analyse de la morphologie des fonds et apportent des connaissances supplémentaires pour l'interprétation de la mosaïque sonar.

L'analyse des conditions de vent a été réalisée sur les mesures enregistrées à une station météorologique installée au CPT Martigues. Trois régimes de vents dominants ont été retenus pour l'élaboration du modèle et l'évolution du panache thermique :

- Les vents de secteur NW à N $\left(320^{\circ}\right.$ à $\left.360^{\circ}\right)$ : vents de terre et Mistral,

- Les vents de secteur E à SE $\left(100^{\circ}\right.$ à $\left.140^{\circ}\right)$ : Levant, Marin et vent de Sud-Est,

- Le temps calme.

Afin d'appréhender la courantologie qui est un facteur prépondérant dans la dispersion des rejets thermiques du CPT Martigues, et pour calibrer le modèle numérique, deux courantomètres (ADCP) ont été immergés. Les données enregistrées ont permis de valider les déplacements des masses d'eau pour les configurations de vent types.

Pour caractériser les masses d'eau, des sondes multiparamètres ont été installées sur des lignes de mouillage. En complément, des profils thermiques ont été réalisés sur 30 stations de mesures réparties sur toute la zone d'étude. Ainsi, pour chaque station et pour chaque condition de vent, un profil a été reconstitué sur la verticale. 
Toutes ces mesures (complétées par des prélèvements de sédiments, une carte biosédimentaire et une qualification de la matière vivante) ont permis de bien qualifier et quantifier la qualité du milieu marin dans l'état initial du projet. Ces données servent de base à l'analyse de l'impact des eaux du système de refroidissement dans l'état projet.

\section{Analyse de l'impact du rejet thermique sur l'environnement marin}

La réalisation d'une étude de caractérisation du rejet en mer du circuit des eaux de refroidissement est nécessaire pour réaliser le volet maritime de l'étude d'impact. L'étude de modélisation concerne la dilution thermique et l'éventuelle recirculation des eaux chaudes rejetées.

Le modèle a été construit sous le système TELEMAC développé par EDF R\&D. L'emprise du modèle tridimensionnel inclut l'amont des darses au Nord, englobe le Grand Etang de Berre et s'étend jusqu'à une limite maritime qui relie la pointe du Port des Tamaris à l'Est à l'embouchure du Rhône à l'Ouest.

Pour chacune des simulations, le modèle prend en compte les phénomènes suivants :

- Échanges thermiques avec l'atmosphère,

- Conditions de marée aux limites maritimes,

- Apports d'eaux douces dans les darses,

- Rejets et prises d'eau industriels à proximité de la centrale dans l'état actuel,

- Rejets et prises d'eau de la centrale dans l'état actuel puis l'état projet.

Après la phase de calibration du modèle sur les mesures en mer réalisées autour de la centrale, le modèle est exploité en intégrant les caractéristiques futures du site.

La situation maximale exceptionnelle (fonctionnement des 2 CCG 24h/24) est présentée ici car elle entraîne des impacts relatifs forcément plus pénalisants que ceux de l'état de fonctionnement réaliste (fonctionnement quotidien des 2 CCG de $6 \mathrm{~h}$ à $22 \mathrm{~h}$ ).

Pour chaque condition hydrométéorologique simulée, l'exploitation du modèle consiste notamment à réaliser une coupe à 1 mètre sous la surface et à 1 mètre au-dessus du fond. L'intérêt de ces coupes est de représenter la température de l'eau dans le panache et de connaître sa distribution jusqu'au fond, zone où les espèces les plus sensibles sont localisées (herbiers, benthos, coralligène, ...).

Les résultats présentés ci-après correspondent à la condition de temps calme. Durant la journée, l'impact du rejet dans la situation future est systématiquement plus faible que dans la situation actuelle, aussi bien à 1 mètre sous la surface libre qu'à 1 mètre audessus du fond (la nuit, la température est bien plus élevée dans l'état futur que dans l'état actuel en raison du fonctionnement permanent de la centrale). Cela est dû à deux facteurs qui dépendent des conditions hydrométéorologiques :

- Soit un débit plus faible même s'il est associé à une élévation de température plus élevée,

- Soit l'ajout d'une pompe de circulation supplémentaire qui permet de revenir au débit de la situation actuelle mais avec une température de rejet bien plus faible. 
Comme dans le cas du Mistral et du vent d'Est, l'impact du panache reste extrêmement limité et n'atteint pas les biocénoses sensibles. En effet, à 1 mètre au-dessus du fond, le panache n'est pas présent et peu susceptible d'impacter les herbiers et le coralligène.
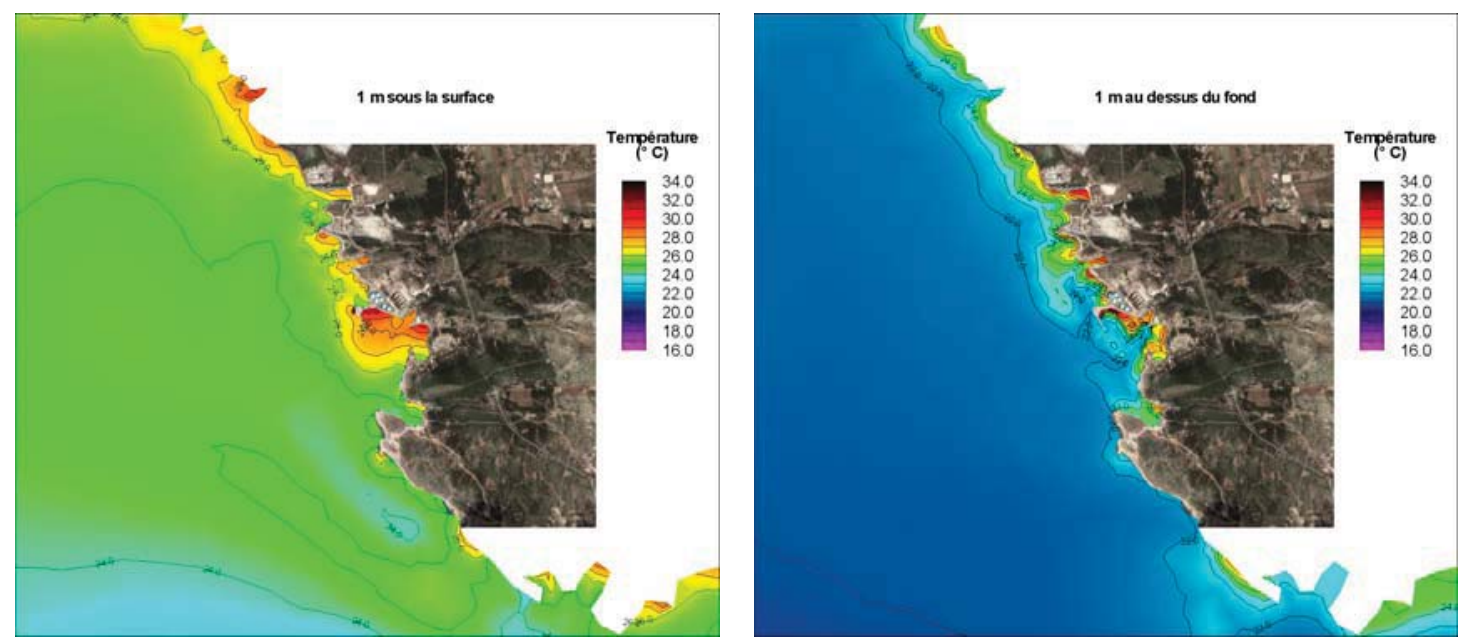

Figure 1. Cartographies de la température des eaux à proximité des rejets du CPT.

\section{Conclusion}

Le projet de la centrale thermique EDF de Martigues consiste à transformer deux des quatre unités de production fioul, en deux unités de production cycles combiné gaz. Ce projet permettra donc de renforcer et de sécuriser le réseau d'alimentation électrique de la zone Provence Alpes-Côte d'Azur, qui consomme aujourd'hui le double de l'électricité qu'elle produit.

Les prescriptions réglementaires nécessitent une analyse de l'état actuel et une description multicritère du milieu marin devant servir de référence à l'évaluation de l'impact du projet.

Une partie des mesures permet aussi de fournir les données nécessaires au calage et à la validation du modèle numérique destiné à évaluer l'état de référence et l'impact, en termes de rejet thermique, sur le milieu de la modification en CCG de deux unités fioul. Le modèle tridimensionnel développé a permis de montrer que le devenir des effluents réchauffés est qualitativement comparable à celui de l'état actuel. Les dimensions en plan du panache des effluents sont même le plus souvent moins importantes que dans l'état actuel et restent très limitées en période critique estivale.

Dans ce sens, l'impact de la mise en fonctionnement des tranches de Cycle Combiné Gaz du CPT Martigues sera donc extrêmement faible, voire même positif, par rapport à la situation actuelle et ce pour une production qui répond à des objectifs d'exploitation croissants. 